\title{
Pure Spastic Paralysis of Corticospinal Origin
}

\author{
C. M. FISHER
}

SUMMARY: This presentation includes six cases of chronic bilateral pure motor hemiplegia, one of these with pathological findings; one clinical case of chronic pare motor quadriplegia and one pathologically-studied case of chronic pure motor paraplegia. These cases may illustrate a spectrum of pure corticospinal disorders that heretofore has not been fully recognized.

RÉSUMÉ: Cet article présente six cas d'hémiplégie motrice pure chronique bilatérale, dont un avec anomalies pathologiques; un cas clinique de quadriplégie motrice pure chronique et un cas autopsié de paraplégie motrice pure chronique. Il est possible qu'il s'agisse ici d'illustrations du spectre non préalablement reconnu de désordres cortico-spinaux purs.

From the Neurological Service of the Massachusetts General Hospital, Boston, Massachusetts 02114, U.S.A.

Presented at the Scientific Session honoring Dr. R. D. Adams, The Ether Dome, Massachusetts General Hospital, May 19-20, 1977.

This study was supported in part by NINCDS, Grant NSOS152.

Reprint requests to Dr. C. Miller Fisher, Department of Neurology, Massachusetts General Hospital, Fruit St. Boston 02114, U.S.A
The nosology of slowly progressive nonfamilial pure corticospinal disorders remains a matter of uncertainty. The best known of these is so-called primary lateral sclerosis with spastic paraplegia. In the literature, references are made to similar states at higher levels of the neuraxis giving rise respectively to spastic quadriplegia, pseudobulbar palsy and spastic bilateral hemiplegia, but well delineated clinical examples are rare and pathological confirmation still rarer.

In recent years we have encountered 6 patients with an unusual neurologic illness in which there slowly developed an extensive spastic paralysis involving legs, arms, face, and tongue without obvious involvement of any system other than the corticospinal tracts and in the absence of a family history of a similar condition. In only one case has a neuropathological study been made and unfortunately the clinical course was complicated by a stroke. These 6 cases which may represent a syndrome not fully recognized are the main subject of the present report. In addition, however, in order to round out the picture, 2 further cases will be described, the first a case of spastic paraplegia with pathological findings and the second an unusual example of prolonged spastic quadriparesis.

\section{HISTORICAL SUMMARY}

Shortly after Charcot and Joffroy (1869) delineated amyotrophic lateral sclerosis, Erb (1875) reported the occurrence of cases with only a corticospinal deficit and no muscular atrophy, referring to the condition as primary lateral sclerosis. Later, Erb (1902) summarized the literature on the subject which consisted of 10 pathologically.studied cases showing severe sclerosis of the lateral columns of the spinal cord from the lumbar to the cervical region and often in addition slight lesions of the tracts of Goll and the direct cerebellar tracts. At least 4 of these cases were examples of the familial variety. Clinically, the patients were described as slowly developing a spastic paraparesis with increased tendon reflexes and Babinski signs without bladder dysfunction or sensory changes. In some cases the tendon reflexes of the arms were enhanced. The age of onset (and the duration of the illness) in the autopsied cases were as follows: 43 years (20 years), 37 (25), 42 (20), 52 (4), 8 (20), $10(20)$ and 1 of unstated age with symptoms for 35 years. Spiller (1904) reported a case (case 6) in which there was isolated spastic paralysis of the legs for $4 \frac{1}{2}$ years. The crossed and uncrossed pyramidal tracts of the spinal cord were distinctly pale. Stark and Moersch (1945) recorded 43 cases of nonfamilial primary lateral sclerosis, none with pathological study. In addition to a spastic paraparesis, 28 had hyperactive reflexes in the arms, 8 had increased tendon reflexes and functional impairment in the arms and 7 showed no involvement of the arms. Wechsler and Brody (1946) described 15 cases of slowly progressive primary lateral sclerosis in which the disease remained in the lower extremities without involvement of the arms or brain stem after follow-up periods of up to 12 years. Only two had sphincter disturbances. Through the years, primary lateral sclerosis has generally been regarded as a variant of amyotrophic lateral sclerosis or motor neuron disease in which anterior horn cell disease appeared later or not at all, the condition lying at the opposite end of the spectrum from progressive muscular atrophy in which cor- 
ticospinal tract sclerosis failed to de velop. Mackay (1963) is representative of this view in his claim that primary lateral sclerosis is simply amyotrophic lateral sclerosis without, as yet. its complement of atrophic change which is always added unless (rarely) death supervenes. He followed 11 cases of primary lateral sclerosis for years and found that 10 eventually showed atrophic changes, only one remaining purely spastic. Not included in his main study were 3 patients whose spastic paresis persisted uncomplicated for 21,14 and 8 years respectively.

An illness closely resembling primary lateral sclerosis and which ran in families was initially described by Strümpell (1886). Apparently it is more frequent, for Paskind and Stone (1933) in 1933 found references to 151 families with more than one member with spastic paraparesis. In the familial illness the predominant clinical findings are spasticity of the legs but there also are instances of involvement of the arms, pes equino-varus, cerebellar ataxia, tremor, dementia, optic atrophy, muscle wasting and scoliosis, indications of a link with Friedreich's ataxia or other hereditary ataxias. Roe (1963) found 176 reports of 250 families with the hereditary form. Schwarz (1952) summarized from the literature 24 cases of familial spastic paralysis in which there were pathological studies. Of the 24 cases, only 7 were typical examples of a pure corticospinal deficit. Pathologically, in these typical cases there were no changes in the internal capsule, cerebral peduncles, pons or medulla. The lateral corticospinal tracts were symmetrically involved in all cases, chiefly at the thoracic level, less at the upper cervical level. The uncrossed pyramidal tract was involved in one half the cases. The fasciculus gracilis was symmetrically demyelinated in the cervical region. It is possible that sporadic primary lateral sclerosis is an isolated manifestation of a familial trait, rather than part of motor neuron disease.

It should be emphasized that in the non-familial group pathological studies are available in only a few cases and in none since 1905. Most cases are from the period before the discovery of the myelopathy of cervical spondylosis and before the routine use of myelography. Also chronic multiple sclerosis, spinal angioma, vascular disease, slowly growing tumor, atlanto-occipital anomalies, and the Arnold-Chiari malformation may not have always been satisfactorily excluded. On the other hand, in response to the claim that all pure corticospinal disease is but amyotrophic lateral sclerosis in evolution, one might ask for more clinical details of the patients who were followed for years and finally developed atrophic changes. How long did the purely spastic state persist before atrophy occurred? Which parts developed atrophy? What was the pace of evolution? Was a pathological study obtained? Such a presentation, which is not available in the literature, would assist the clinician in classifying his cases.

Compared with that of primary lateral sclerosis, the history of the other pure corticospinal disorders is brief. Chronic progressive spastic quadriplegia is vaguely alluded to in discussions of primary lateral sclerosis in which the arms become involved, but no specific case has been recorded. Chronic progressive spastic bulbar paralysis has been described by Aird (1962) as an illness occurring in early middle life, frequently in association with slight spasticity of the limbs and hyperactive deep reflexes and is classified as a variant of amyotrophic lateral sclerosis. The entity of pure spastic dysphonia or dysarthria is not clearly set forth in the literature. Chronic progressive double hemiphegia is represented by a single but striking case report. Buzzard and Barnes (1906) reported the case of a woman, aged 53, who for 10 years had a slowly progressive illness beginning with uncontrollable laughter and progressing to dysarthria and finally weakness of the legs. The patient walked with a stiff gait scraping the floor, rapid movements of the fingers and tongue were slowed, there was no muscular atrophy, the reflexes were brisk and the plantar responses were extensor. Inappropriate spasms of laughter were prominent. There was no history of a stroke. The patient died suddenly after being observed in the hospital for about 4 months. On neuropathological examination the pyramidal tracts showed in the myelin stain sclerosis and pallor throughout their length - the anterior $2 / 3$ of the internal capsule, the fronto-pontine fibers of the cerebral peduncles, medullary pyramids and posterior portions of the lateral columns of the spinal cord from the cervical to the sacral levels. The ganglion cells of the cortex may not have been as large as usual. No other part of the brain was involved and the anterior horn cells of the spinal cord were normal. There was severe arteriosclerosis of the penetrating arteries of the brain and the authors concluded that their case was one of chronic progressive double hemiplegia secondary to the ischemid of vascular disease rather than of a primary nature. The illustrations are particularly lucid. The crossed corticospinal tracts were not involved. Swank and Putnam (1943) stated that in one of their 21 cases of primary lateral sclerosis pseudobulbar palsy with emotional lability developed later. Chronic progressive single or unilateral hemiplegia has not been described as an entity.

CASE MATERIAL

A. Chronic progressive spastic double hemiplegia - chronic bilateral pure motor hemiplegia - Chronic progressive bilateral spinobulbar spasticity.

The six cases described below resemble one another sufficiently to suggest they represent a recognizable clinical syndrome. A somewhat serious weakness lies in the fact that only one case has been studied pathologically and in that case the occurrence during the course of the illness of a pontine hemiplegic stroke complicated both the clinical and the pathological aspects. In the remaining five cases, common neurological disease has been largely excluded, but the possibility that atypical processes, for example, unremitting multiple sclerosis, were the cause of 
the syndrome in some cases is a concern. Only further pathological studies will allow more accurate conclusions. In no case was the course of the illness marked by exacerbations and remissions.

\section{Case 1}

The patient began to have stiffness of the legs, poor balance and difficulty walking at the age of 67 . Within 10 months he began to use crutches. After 17 months the arms became progressively weaker. The thighs and legs ached anteriorly and posteriorly. A myelogram after 15 months was negative. At the time of onset. the patient had developed an acute low backache while lifting. There was a past history of migraine headaches and of a suprapubic prostatectomy at the age of 62. Examination at the age of 68.20 months from onset. showed an exceedingly severe spastic paraplegia with the legs in rigid extension. The arms were slow and weak. The tendon reflexes were abnormally brisk and the plantar responses were extensor. The cranial nerves and sensation were normal. There was no dysarthria. The blood pressure was 175 systolic. The patient gradually got worse and at the age of $69,2 \frac{1}{2}$ years from the onset, suddenly developed a severe left hemiplegia associated with dysarthria and dysphagia. Within 1 week he began to walk with assistance but he never quite recovered to the prehemiplegic level. After a period of further improvement, the quadriparesis grew gradually and steadily worse. The low backache continued. One episode occurred in which the weakness of the arms and legs markedly increased, with the result that the patient could scarcely move. The exacerbation had subsided by the next morning. Examination at the age of 70 (9 months after the hemiplegic episode) showed spastic dysphonia and severe dysarthria and speech was described as a barely articulated monotone. There were spasms of inappropriate rapidly reversing laughter and crying. Movements of the face, tongue and all limbs were slow. The limbs were severely spastic. Using a metal-framed walker, the patient advanced each foot a few inches at a time. The tendon reflexes were brisk and the plantar responses extensor. Sensation was normal. The ocular movements were normal without nystagmus. The mind was well preserved. The optic discs were sharply outlined and of normal color. There was no cerebellar ataxia. There were no grasping or sucking reflexes. The Romberg test was negative.

The hemoglobin was $15.0 \mathrm{gms}$. and the WBC 8,100 . A urinalysis was normal. There was gastric free acid. The CSF was under a pressure of $123 \mathrm{~mm}$. and contained $38 \mathrm{mg} . \%$ of protein and no WBC. The colloidal gold test was 0012210000 . A ${ }^{64} \mathrm{Cu}$ scan was negative. An EEG was normal. The patient lived in a nursing home for 2 more years, dying at the age of 72,5 years from onset, of a myocardial infarction. There were no further recognizable strokes. The arms and legs remained spastic but the patient was able to feed himself and move a wheel chair despite the marked spasticity of the arms. His intelligence was preserved. He could not speak because of articulatory difficulty and communicated by writing notes.

Neuropathological Findings: Grossly there was an old infarct in the right basis pontis at the junction of the upper $1 / 3$ and lower $2 / 3$ of the pons. The infarct consisted of 4 small cavities lying in an anteroposterior line in the paramedian area a few $\mathrm{mm}$. from the midline. The right medullary pyramid was reduced in size. These were the only gross abnormalities. Sections were prepared and stained by several methods including Nissl, myelin. Scharlach R, Holzer and Bodian. On microscopic examination the internal capsules and midbrain peduncles appeared normal. There were no scattered lesions to suggest multiple sclerosis. The giant pyramidal cells of the motor cortex were probably reduced in number. In the pons the series of old small cavitating infarcts in the right paramedian area were obvious. Both medullary pyramids were pale in the myelin stain, the right more than the left. (Figure 1) In Cajal and Bodian preparations large fibers were greatly reduced in the pyramids but gliofibrillar material was not increased. In the Scharlach $R$ stain there were no fatty macrophages but myelin was less prominent than in control cases. The cerebral cortex, putamen, globus pallidus, thalami, substantia nigra and brain stem tegmentum were normal.

In the spinal cord there was pallor in the region of the lateral corticospinal tracts of the entire cord from the upper cervical region to the lumbosacral level. (Figures 2 and 3 ) The right anterior uncrossed corticospinal tract was pale but the left stained well. The anterior horn cells at all levels were preserved in number and structure. The anterior rootlets in the cervical and lumbosacral regions were not demyelinated.

Comment: The pontine paramedian infarct accounted satisfactorily for the patient's acute stroke three years before death. It probably was also responsible for the greater pallor of the right medullary pyramid. It would not account for the striking pallor of the lateral corticospinal tract bilaterally. Furthermore, the clinical course was a slow gradual bilateral progression punctuated only once by a sudden acute onset from which good recovery occurred in keeping with a vascular insult. The preservation of power in the limbs late in the illness and the maintenance of the intellect were noted in the other cases. It was therefore concluded that vascular disease was only a complicating factor and the true nature of the illness was of an entirely different type.

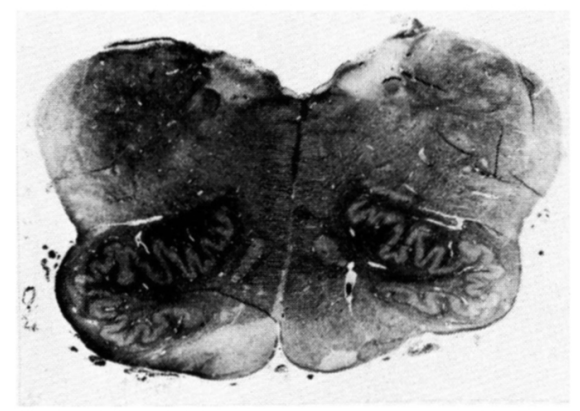

Figure 1-Case 1. Section of medulla oblongata (Cajal with myelin counter stain) showing marked pallor of right pyramid. lesser pallor of the left.

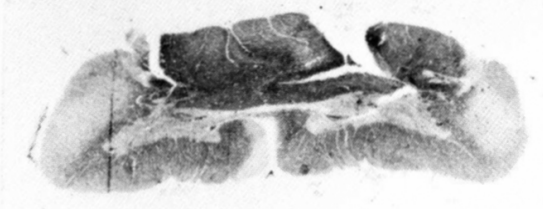

Figure 2-Case 1. Section of cervical spinal cord (myelin stain) showing pallor of corticospinal tracts lateral and anterior on the right side. lateral only on the left side.

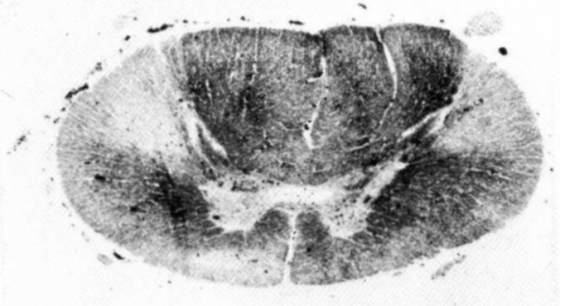

Figure 3-Case 1. Section of thoracic spinal cord (myelin stain) showing pallor of corticospinal tracts. 


\section{Case 2}

The patient, a male engineer, at the age of 61 first noted loss of coordination of the legs and inability to run as he ordinarily would. He was no longer able to balance on his bicycle. Soon he began to drop things from his hands and finger movements became slowed. The disability in the arms and legs slowly and gradually progressed. He tended to tire easily and fell frequently. At the age of 65 , slurred speech was first noted. He said his speech was as if he had to force it out. At the age of 66 he could walk on a treadmill for $\mathbf{4 0}$ minutes at $2 \frac{1}{2}$ miles per hour and at the age of 67 only 21 minutes at 2 miles per hour. He thought that at the beginning of his illness the left leg and arm were more affected than the right. When seen at the age of 67,6 years from the onset, he had recently noted the appearance of slight dysphagia, a lowered pitch of the voice and nocturnal cramps in the legs. Bladder and bowel function were normal. A definite lability of laughter and crying had appeared.

On examination (age 67) there was a definite dysarthria with an element of spastic dysphonia. He could easily be understood. There was no nystagmus. La, la, la was greatly slowed. There was a bilateral corneal-pterygoid reflex. All limbs were moderately spastic, left more than right. Motor power was approximately normal when the patient was given time to make a full exertion. He could step up on an $8^{\prime \prime}$ stool. He could support himself on the heels or toes. There was no atrophy or fasciculation. The tendon reflexes were all brisk and a Babinski response was present bilaterally. The jaw jerk was absent. Sensation was intact to pinprick, vibration, touch and joint position sense. There was no tremor or cerebellar ataxia. $\mathrm{He}$ swayed $2^{\prime \prime}$ on the Romberg test. The patient walked with a slow, spastic gait, scraping the toes, giving the appearance of wading in water up to the mid-riff. There was lability of laughter and lacrimation.

An extensive laboratory investigation including myelography, arteriography and CSF examination was normal.

When reexamined 2 years later (age 69) all symptoms had progressed. People had difficulty understanding his speech. Swallowing was more impaired. He could walk on a treadmill for only 2 minutes at $1 \frac{1}{2}$ miles per hour. At night the legs prickled as if a thousand needles were being jabbed into them. Nocturnal cramps had continued. The neurological signs had changed only in degree. Speech was more dysarthric and slowed ( 3 words per second), the face moved symmetrically. Motor power was still approximately normal. Maximum rapidity of movements was greatly decreased. Intelligence was preserved. In the next 3 years there was a further slow decline, but the patient was still able to indulge in his hobbies 11 years from the onset of the illness.

\section{Case 3}

The patient, a male sales-manager, first noted the onset of a progressive clumsiness of the legs and arms at the age of 61 . For the first
2 years, the left arm and leg were predominantly affected, while in the 3rd and 4th years, the right side became involved. In the 5 th year speech became slurred to an extent, it made the patient "hang his head in shame". The legs were stiff and balance so poor, the patient often fell. On going down stairs, his knees were on the verge of buckling. Cramps occurred in the leg muscles at night. A definite lability of laughing and crying had appeared. At the onset of the illness, the left index finger was numb and this persisted. Bladder and bowel function were preserved. The patient had become stooped and feeble. Intelligence was preserved.

On examination at the age of 66 (in the 5 th year) there was only a slight dysarthria. There was no nystagmus. The optic discs were normal. The patient gave the general appearance of rigidity of the trunk and neck. La, la, la was performed slowly. The jaw jerk and facial reflexes were very brisk. Motor power in the arms and legs was approximately normal when the patient was given time to make a full effort. There was no atrophy or fasciculation. There was moderate spasticity in all limbs with increased tendon reflexes and a Babinski sign bilaterally. Sensation was intact to pinprick, touch, joint position sense and vibration. The gait was slow, jerky and spastic. The Romberg test was normal.

Extensive laboratory investigation including myelography, cerebral arteriography, CSF, EEG and EMG were normal.

There was slow progression in the next 5 years without new neurological findings.

\section{Case 4}

The patient, a woman, suddenly at the age of 68 developed a left hemiplegia involving face, arm and leg from which recovery was excellent in about 3 weeks. At the age of 69 she complained of slurred speech, hoarseness and a gradual loss of voice with talking. Examination showed slight dysarthria, slowness on the la, la, la performance and weakness in placing the tongue in each cheek. Power in the right hand grip was $7 / 10$, in the left $8 / 10$. In the next 3 years speech became gradually and slowly worse and walking more difficult without further recognizable episodes of cerebral ischemia. On examination at the age of 72 speech was slurred almost to the point of being unintelligible and the tongue could not be placed in either cheek, but there was no atrophy. The mouth hung open. There was no buccal-lingual dyspraxia. There was no nystagmus. Swallowing was a little difficult. The jaw jerk was $4+$. There was a tendency to inappropriate grinning. The facial jerks were $3+$. Power in the arms and legs was approximately normal. The limbs were spastic, the tendon reflexes all very brisk and Babinski and Hoffman signs were present bilaterally. Finger tapping was greatly slowed. There was no tremor or cerebellar ataxia. There was no definite sensory loss except for decreased vibration below the mid-shin. The patient walked slowly on a wide base, using a metal-framed walker. She could not stand by herself. Mind and judgment were unimpaired. The blood pressure was $200 / 90$.
In the next 3 years the patient became progressively more helpless. On examination at the age of 75 she was unable to speak at all. There was moderate dysphagia. She communicated by means of laborious writing. The mouth hung open and the lips were held in a position of constant smiling. There was no nystagmus. Eye closure was strong, whereas power of lip closure was zero. The lips moved well in laughter. There was a definite inappropriate lability of laughing and crying. The tongue moved a few $\mathrm{mm}$. from side to side. Tone was moderately increased in all limbs and the tendon reflexes were brisk. There was a bilateral Babinski sign. When the patient was given time to exert full effort there was good power in all limbs. Sensation was unchanged from previously. There was no tremor or cerebellar ataxia. The patient knew the date and the stock market quotations of the day. The CSF was under a pressure of 170 and contained $32 \mathrm{mgs}$. of protein and no WBCs. The gamma globulin was $2.5 \%$. A pneumoencephalogram showed a ventricular span of $40 \mathrm{~mm}$. and slight widening of the quadrigeminal cistern, suggesting mid-brain atrophy. During the following $1 \frac{1}{2}$ years there was a further decline and at the age of 76 the patient sat with her head hanging and chin on chest. The mouth hung open $1^{\prime \prime}$. The patient smiled broadly. The tongue protruded only to the line of the teeth. The patient drooled and made unintelligible laryngeal sounds. There was no nystagmus and the patient could not turn her eyes from side to side on spoken command. She could very slowly bring a coffee cup or a napkin to her mouth. She wrote slowly at about one letter every 3 seconds. She chewed and swallowed slowly but seldom choked. There was a remarkable clasp-knife spasticity of all limbs and a Babinski sign bilaterally. There was no tremor or cerebellar ataxia. Sensation in the fingers and toes was within normal limits for the patient's age. She was continent without urgency. The left leg involuntarily extended at the knee for about 3 seconds at a time. The patient died at the age of 76 from gastroenteritis and perforation of the bowel.

\section{Case 5}

The patient, a woman journalist, noted at the age of 66 that her speech was slurred and less rapid whereas normally she spoke "like a cannonball." The impairment gradually progressed and at the time of examination 3 years later at the age of 69 she said she spoke "like a 3-year-old child separating the syllables." Her throat became tired when she talked. Speaking became an effort and she had to "push" her voice which seemed to be higher pitched. At times there was stridor on inspiration. In the previous few months inappropriate lability of laughter and crying had appeared. Six months before examination the right eyelid drooped slightly. For 6 weeks there had also been difficulty and pain in swallowing. Occasionally she saw slightly double. The arms and legs were reported as normal but typing had become inaccurate and slow and whereas the patient had formerly swum a $1 / 2$ mile daily, she now could scarcely swim 6 strokes. Climbing the stairs was dif- 
ficult. Six weeks before, she developed back pain at the T12 to L2 level. In general, the patient thought she had slowed up greatly in the previous 2 years.

Neurological examination showed slow, indistinct, slurred, precise speech. The extraocular movements were full without nystagmus. The orbicularis oculi was strong bilaterally but there was a right lower facial weakness. La. la. la and me, me. me sounds were greatly slowed. Serial expiration was difficult. The tongue was strong and free of atrophy. The palate elevated poorly. There was no obvious ptosis. The jaw jerk was $3+$ and the facial reflexes were enhanced. Power in the arms and legs was approximately normal. The tendon reflexes were increased, particularly on the right side, and the plantar responses were flexor. Sensation to pinprick, vibration. touch and joint position sense was normal. Rapid alternating movements were well performed. The Romberg test was negative and the patient could walk tandem in a straight line. Her intellect was preserved. The CSF was under a pressure of $22 \mathrm{~mm}$. of water and contained $72 \mathrm{mg} . \%$ of protein.

In the following year speech became more impaired and rapid movements of the fingers and toes became slowed. All tendon reflexes were brisk but the plantar responses were flexor. There was slight weakness in the right upper extremity. There was no tremor or cogwheeling.

Another year later, at the age of 71 , speech had become almost unintelligible. The right arm had become weak and of little use. The left arm could not be used for fine tasks but served for eating and dressing. Gait was slow and the patient had fallen many times. Dysphagia was slowly advancing. There had been no tingling or numbness. Hearing was normal. Her mind was intact. The extraocular movements were full without nystagmus. There was great difficulty in moving the tongue from side to side. There was difficulty in turning the eyes from side to side on command. There was a moderate right facial weakness. Jaw closure was weak. The tongue moved slowly but was not atrophic. The jaw jerk was increased.

Motor power was good in resisting passive movements but the left arm and leg were stronger than the right. All movements were greatly slowed. There was a clasp-knife spasticity of all extremities. The tendon reflexes were hyperactive and there was a Babinski sign bilaterally. Sensation was intact to pinprick, vibration, touch, joint position sense and stereognosis. There was no tremor, cerebellar ataxia or cogwheeling. The patient walked with 14" slow shuffling steps. The Romberg test was negative. An EEG was normal. An EMG showed no evidence of lower motor neuron disease. Cortical function tests showed retention of her mental ability. The blood pressure was $122 / 76$.

The patient committed suicide 3 months later.

\section{Case 6}

The patient, a woman, first noted slurred. slow'ed, deliberate speech at the age of 50 . During the next year she began to drag the right leg and in the following year the left. Thereafter, there was a slow gradual progression of gait impairment without remission. At the age of 56. handwriting became slowed. buttoning difficult and rapid movements of the fingers impaired. Dysphagia occurred on drinking or eating too quickly. On neurological examination at the age of 60.10 years from the onset. speech was slow and slurred and had a quality of spastic dysphonia. It could be readily understood. La, la, la and me, me, me were greatly slowed. The patient walked slowly scraping the toes on the floor in typical spastic fashion. She could take steps by herself but preferred to hold on. Power in the limbs was within normal limits. both proximally and distally when the patient was given sufficient time to exert full force. She could step up on an $8^{\prime \prime}$ stool with either leg. The arms were moderately spastic, the legs much more so. The tendon reflexes were all extremely hyperactive and Babinski signs were present bilaterally. Ankle clonus was present bilaterally. There was no atrophy of muscles. The voice was hoarse but the patient thought this was normal. The jaw jerk was brisk but the facial reflexes were not increased. There were slight mirror movements of the left arm when the right arm was pronated and supinated. Sensation was intact to pinprick. vibration. touch and joint position sense in the fingers and toes. There was no tremor or cerebellar ataxia. The most rapid movements of the limbs were greatly slowed. There was no nystagmus. The optic discs were of good color. The intellect was preserved. There was inappropriate ease of laughter and crying. There was no urgency or incontinence of bowel or bladder. There had been no diplopia. There had been no numbness of the fingers or toes except occasionally for a few minutes.

Numerous laboratory tests including EEG. EMG, carotid and vertebral angiography, CSF and brain scans were all normal.

\section{COMMENT}

The cases included 3 males and 3 females, ranging in age at onset from 50 to 69 years, 5 being in the $60 \mathrm{~s}$. In all 3 women the illness began with dysarthria and advanced to involvement of the limbs, whereas in the men, the opposite was the case. The tempo of the gradually progressive process was variable and led to severe disability in the following periods respectively -5 years, 8 years, 7 years, 6 years, 5 years and in case 6 the disability was only moderate after 10 years. The final picture was stereotyped - spastic quadriparesis, hyperactive tendon reflexes, a Babinski sign bilaterally, severe dysarthria and moderate dysphagia with preservation of power to resist passive motion. Lability of laughing and crying occurred in all cases. There was no sensory loss, cerebellar incoordination, nystagmus, tremor, impairment of eye movements, bowel and bladder dysfunction. muscle atrophy or fasciculation. The intellect was preserved. There was no clinical or laboratory evidence of anterior horn cell involvement. Intrinsically, the illness does not appear to be fatal. and the causes of death in the 3 cases dying were myocardial infarction, perforation of the bowel and suicide respectively.

Judging from the pathological findings in case 1 , there is a loss of fibers and myelin in the corticospinal system bilaterally. traceable from the lumbar level upwards through the spinal cord to the level of the medullary pyramids but not higher. The fasciculus gracilis may have been a little pale at the cervical levels. In the available sections of the cerebral cortex the giant cells of Betz were probably reduced. The anterior horn cells of the spinal cord were intact.

Regarding the nature of the illness the process corresponds to amyotrophic lateral sclerosis in which anterior horn cell disease is missing. However, if the disease were a variant of amyotrophic lateral sclerosis, one might expect to encounter intermediary forms in which amyotrophy is added to spasticity after a period of several years, but this has not been our experience. The relative benignity of the process would indicate that it is lower motor neuron disease that is the limiting factor in survival in amyotrophic lateral sclerosis. There is no advantage in forcing the illness into the category of amyotrophic lateral sclerosis and indeed a good case could be made for regarding it as a variant of primary lateral sclerosis. The illness faintly resembles progressive supranuclear palsy but lacks supranuclear ophthalmoplegia, axial dystonia in extension, rigidity of the limbs and dementia. It might be added here that in the 6 cases, urinary and blood screening tests for amino acid abnormalities were negative.

\section{B. Chronic progressive spastic quadriplegia.}

There is only one patient in this subcategory, an unusual case of 
progressive quadriparesis in which a severe disability developed over a period of 6 years and then remained stationary for the next 9 years.

\section{Case 7.}

A male civil engineer first noted the onset of progressive difficulty walking at the age of 50. The right leg was involved first and the left. leg about 3 months later. After 9 months he began to use a cane. After 18 months the arms became involved, in that writing was difficult. All limbs became progressively slow, stiff and weak. At the time of neurological examination $31 / 2$ years from the onset, the patient could stand but scarcely move the legs. The right arm was slightly useful, the left arm almost useless. Flexor spasms of the legs occurred at night and on standing the legs tended to scissor. Urinary bladder function varied from urgency and occasional incontinence on the one hand to comfort for as long as $\mathbf{2 0}$ hours. Bowel and sexual functions were normal. Shortness of breath occurred after talking. There had been no pain, numbness, dysarthria or dysphagia. The patient reported a striking variation in the disability and in the earlier stages sometimes he was able to walk fairly well or climb stairs, for approximately 4 hours at a time. At first the right leg would become heavy after walking 400 meters with full recovery after 20 minutes of rest. His voice had reduced volume.

On examination all limbs were extremely spastic. There was, however, surprisingly good strength in resisting passive movements at the shoulders, elbows, wrists, hips and knees. The hand grips were approximately $7 / 10$ on the right and $6 / 10$ on the left. The right hand was useful for gripping a cane. The tendon reflexes were all greatly increased and there was a bilateral Babinski response. The jaw jerk was $1+$ and there was no suck or grasp response. Sensation was intact to pinprick, vibration, touch, joint position sense and cold in the upper limbs and in the lower limbs except that vibration was felt for $11 / 20$ seconds at the right great toe and was absent at the left great toe. The knee jerks were $4+$. the ankle jerks $2+$. Shrugging the shoulders was greatly slowed. Periodically there occurred strong extensor spasms of the legs.

Cranial nerve function was approximately normal. The pupils were $3 \mathrm{~mm}$. and reacted to light. The extraocular movements were full without nystagmus. The fundi and discs were normal. The right side of the face was questionably weak. The tongue was strong without atrophy. Rapidity of tongue movements was not slowed. There was no cerebellar ataxia or tremor. Intelligence was intact. The blood pressure was $120 / 70$.

The laboratory tests were as follows: Hemoglobin 14.2, white blood count 9,100 . Urinalysis normal. Sedimentation rate 12 . Urine and blood tests for abnormal amino acids were negative. An electromyogram showed no fibrillations or fasciculations in arms or legs. A cervical myelogram was normal. The CSF was clear and colorless, contained no WBCs and the protein was 50 $\mathrm{mg} . \%$.
The patient slowly became more disabled in the following 2 years. Thereafter, however, although almost completely paralyzed in the arms and legs, he remained on a plateau enjoying otherwise good health with preservation of mind, speech and swallowing. At the age of 65,15 years from the onset, he was being cared for in a chronic hospital where he was occupied reading the works of Sir Winston Churchill utilizing an electronic page-turner. (We are indebted to Dr. John Armstrong for the follow-up information that no muscle attrophy has occurred.)

Comment: Although only the clinical details are known the illness appears to be unique and not easy to fit into the ranks of any known neurological disease. Is it doubly atypical amyotrophic lateral sclerosis with neither muscle atrophy nor bulbar involvement? This is conceivable. Or is it "primary lateral sclerosis" with higher spinal cord involvement than in the reported cases. Strümpell (1901) referred to cases of spastic paralysis of the arms and legs at an advanced stage associated with a slight muscle atrophy and thought the process merged with amyotrophic lateral sclerosis. However, cervical spondylosis was not recognized in that era.

If the present syndrome and primary lateral sclerosis are related to amyotrophic lateral sclerosis, it might be anticipated that cases of amyotrophic lateral sclerosis would be found in which there is uppermotor-neuron involvement of only the lower extremities, sparing the arms and tongue, or of the lower and upper extremities, sparing the tongue. Yet pathologically, demyelination of the internal capsule occurs in almost all cases of amyotrophic lateral sclerosis (Lawyer and Netsky, 1953). This case of pure quadriplegia illustrates again that, as in primary lateral sclerosis, corticospinal tract disease may "burn itself out" following which survival is prolonged.

\section{Pure spastic paraparesis.}

The case to be described resembles primary lateral sclerosis in many ways but is atypical in the age of the patient and the short duration of the process at the time of death. A cervical myelogram ostensibly excluded compression myelopathy. The pathological study is the only one reported in many years.

\section{Case 8}

The patient, a retired abstemious machinist, noted the insidious development of increasing stiffness of the legs at the age of 77 . At the time of onset, there was a dull aching pain in the paravertebral region on the right side between the neck and the mid-thoracic level and a few weeks later a similar pain on the left side. Examination 6 months from the onset showed much difficulty in getting to the feet and a striking slowness in walking, which was a great effort. The gait was stiff-legged and of the circumduction type. The cranial nerves were normal and there was no nystagmus or dysarthria. The arms were normal except for brisk tendon reflexes. The legs were essentially normal in strength despite great spasticity. There was no cerebellar ataxia. The sphincters were intact. Sensation was normal. Using a biothesiometer, vibration was slightly reduced below the knees. Intelligence was preserved. The blood pressure was $110 / 70$.

The hematocrit was 41 and the WBC 6,850 . The sedimentation rate was 11 . Urinalysis normal. A gastric aspirate contained free acid. The CSF dynamics with the head in neutral, extended and flexed positions were normal. The CSF was clear, contained $63 \mathrm{mg} . \%$ of protein and the colloidal gold was 1122211000 . A myelogram showed moderate cervical spondylosis without cord compression. An EMG of both legs and the left arm was normal. Skull $x$-rays were normal. An EEG was slightly abnormal. Hinton tests of the blood and CSF were normal. The jaw jerk was $1+$. Flexor spasms of the legs were troublesome. Frequency and urgency of urination began, without incontinence.

During the following year, a slow gradual progression of the difficulty in walking occurred but the patient was still able to walk with a metal-framed walker. Flexor spasms continued to occur both day and night. Six months after discharge, the patient noted a prickling numbness of the fingers of the right hand, beginning in the tip of the thumb and index finger and progressing towards the 5th finger. The tingling involved only the distal two phalanges. Buttoning and writing were difficult because of the sensory impairment. Because of bladder incontinence an indwelling Foley catheter was inserted. Examination $1 \frac{1}{2}$ years from the onset showed increased stiffness, spasticity and disability. The tendon reflexes in the arms were $2+$, the knee jerks $3+$ and there was a bilateral Babinski response. Pinprick and light touch were diminished in the fingers of the right hand but vibration and position sense were intact. It was clear that there had been progression in the previous year.

Two months later the patient died from chronic bowel obstruction which proved to be due to a volvulus.

Neuropathological Findings: Sections were prepared of the sensorimotor cortex, several other regions of the cerebral cortex, thalamus, internal capsule, midbrain, pons, medulla and 27 levels of the spinal 


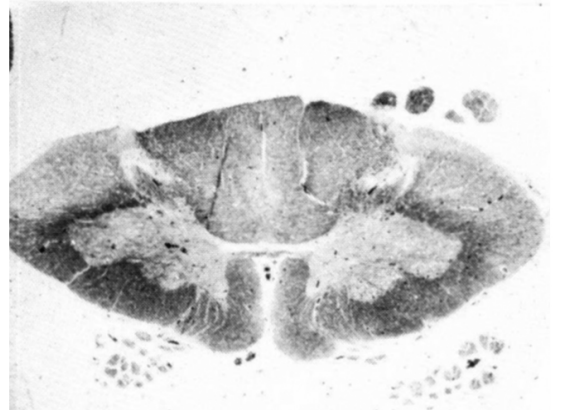

Figure 4-Case 8. Section of cervical spinal cord (myelin stain) showing faint pallor of lateral corticospinal tracts.

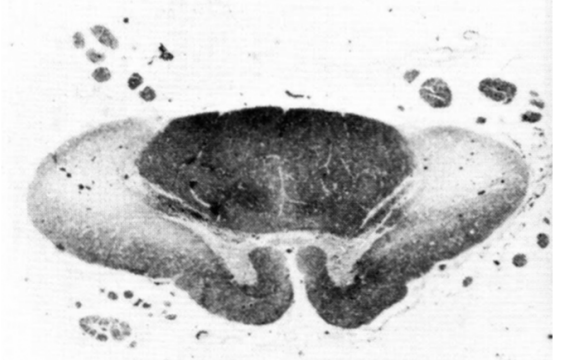

Figure 5-Case 8. Section of thoracic spinal cord (myelin stain) showing marked pallor of corticospinal tracts and a more generalized slight pallor of the entire cross-section except the posterior columns.

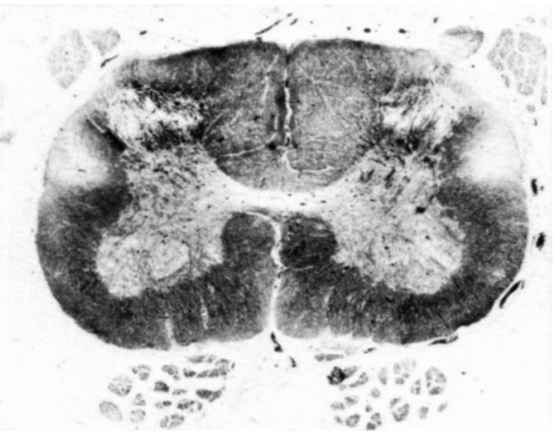

Figure 6-Case 8. Section of lumbar spinal cord (myelin stain) showing pallor of corticospinal tracts.

cord. Staining methods included Nissl, myelin, Bodian, Holzer and Scharlach R. There was no abnormality of the cerebrum and brain stem. At the $\mathrm{Cl}$ level there was a small but definite zone of pallor in the posterior portion of the lateral column in the region of the corticospinal tract bilaterally. At the C8 level the posterior part of the lateral compartment was extensively pale. (Fig-

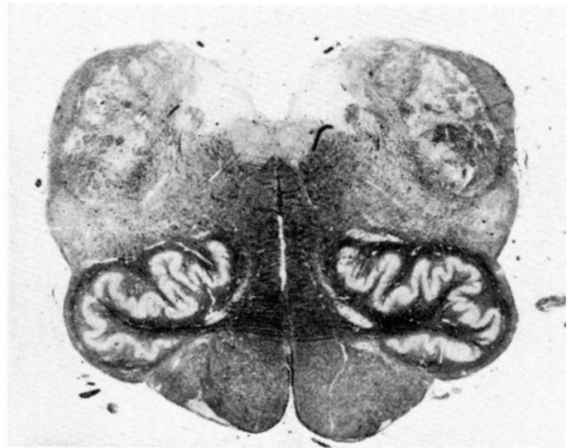

Figure 7-Case 8. Section of medulla oblongata (myelin stain) showing normal staining of pyramids.

ures 4, 5, 6, 7) At T6 this was even more pronounced. The uncrossed corticospinal tracts were intact. At L2, L4 and S1 levels a well demarcated zone of corticospinal pallor was obvious on myelin stains. The anterior horn cells and the anterior rootlets at all levels were well within normal limits. Scharlach $\mathrm{R}$ staining disclosed a scanty collection of fatty macrophages at the $\mathrm{C} 8$ level and none higher. At the TI level bilaterally in the area of the myelin pallor, there was an abundance of Scharlach R-positive fat-filled macrophages. In addition, there was an abundance of what appeared to be free Scharlach R-positive-material collected aroung small blood vessels. A similar appearance was present throughout the entire thoracic cord and disappeared at the L2 level. The great number of fatty macrophages at the $\mathrm{T} 1$ level compared with their scarcity at C8 was most striking and suggested a focal process acting in this region of the spinal cord, for example, cord compression. However, there was no evidence of ascending degeneration in the spinal cord, the uncrossed corticospinal tracts were intact and in life a cervical myelogram had been negative. There was no evidence of multiple sclerosis.

Comment: The slow gradually progressive development of a severe spastic paraplegia in the absence of a sensory deficit makes the case a good candidate for the diagnosis of primary lateral sclerosis. Electromyographic studies showed no evidence of anterior horn cell disease. Numbness of the fingers of the right hand and increased tendon reflexes were the only signs of involvement of the arms. Involvement of bladder function was atypical.

The pathological findings of selective demyelination of the lateral corticospinal tracts below the C 8 level and slight demyelination of the fasciculus gracilis have been reported in two cases of familial primary lateral sclerosis (Strümpell, 1901; Bischoff, 1902) and one case of the sporadic form (Dejerine and Sottas, 1896). The possibility that the combination represents descending and ascending degeneration due to a cervical cord lesion cannot be discarded entirely despite the absence of a spinal block and cord compression by myelography. However, if compression of the cervical cord occurred evidence of it should have been observed in at least one level of the cervical cord.

We have had a number of similar cases of pure spastic paraparesis of undetermined origin, some with questionably significant spondylosis in which decompression did not change the downward course of the illness and in some with no spondylosis at all. Probably in future cases, more attention should be directed to the crucial matter of an obscure mechanical insult in the cervical region.

The preservation of motor power in the legs despite great spasticity is commonly referred to in the literature. However, there are cases with a more or less flaccid pure motor paraparesis without fasciculation, atrophy or electromyographic signs of anterior horn cell disease, but pathological study of such a case has not been reported in the literature.

\section{CLOSING REMARKS}

Most of the important points have been discussed in the introduction or where appropriate in the three different sections. Chronic bilateral pure motor hemiplegia does not appear to have been described previously as a primary disorder and no clear reference to chronic pure motor quadriplegia has been found. These two states and the category of primary lateral sclerosis appear to form a kind of natural spectrum of pure 
corticospinal disease but further case reports and more pathological studies are necessary to test this proposal. Their possible relationship to amyotrophic lateral sclerosis is obviously of theoretical interest.

\section{REFERENCES}

AIRD, R. B. (1962). Clinical Neurology, A. B. Baker, Editor. Hoeber-Harper, Chapter 38, P. 1743.

BISCHOFF, E. (1902). Pathologischanatomischer Befund bei Familiärer infantiler spastischer spinalparalyse. Jahrbuch fur Psychiatrie, Wien, 22, 109-127.

BUZZARD, E. F. and BARNES, S. (1906). A case of chronic progressive double hemiplegia. Review of Neurology and Psychiatry, 4, 182-191.

CHARCOT, J. M. and JOFFROY, A. (1869). Deux cas d'atrophie musculaire progressive avec lésions de la substance grise et des faisceaux antérolatéraux de la moelle épinière. Archives de Physiologie Normale et Pathologique, Paris, 2, 354-367, 629-649, 744-760.

DEJERINE, J. and SOTTAS, J. (1896). Sur un cas de paraplégie spasmodique acquise. Archives de Physiologie Normale et Pathologique, 8, 630-639.

ERB, W. H. (1875). Uber einen wenig bekannten spinalen Symptomen-Complex. Klinische Wochenschrift, 12, 357-359.

ERB, W. H. (1902). Spastic and syphilitic spinal paralysis. Lancet, 2, 969-974.

LAWYER, T. and NETSKY, M. G. (1953). Amyotrophic lateral sclerosis. Archives of Neurology \& Psychiatry, 69, 171-192.

MACKAY, R. P. (1963). Course and prognosis in amyotrophic lateral sclerosis. Archives of Neurology, 8, 117-127.

PASKIND, H. A. and STONE, T. T. (1933). Familial spastic paralysis. Archives of Neurology \& Psychiatry, 30, 481-500.

ROE, P. F. (1963). Hereditary spastic paraplegia. Journal of Neurology, Neurosurgery and Psychiatry, 26, 516-519.

SCHWARZ, G. A. (1952). Hereditary (familial) spastic paraplegia. Archives of Neurology \& Psychiatry, 68, 655-682.
SPILLER, W. G. (1904-1905). Primary degeneration of the pyramidal tracts; a study of eight cases with necropsy. University of Pennsylvania Medical Bulletin, 17, 390-395. 407-414.

STRÜMPELL, A. (1886). Ueber ein bestimmt Form der Primären combinierten Systemerkrankung des Rückenmarks. Archiv fur Psychiatrie und Nervenkrankheiten. Berlin, 17, 227-238.

STRÜMPELL, A. (1901. Ueber Hereditäre spastische spinalparalyse. Neurologisches Centralblatt, 20, 628-629.

STARK, F. M. and MOERSCH, F. P. (1945). Primary lateral sclerosis. Journal of Nervous and Mental Disease, 102, 332-337.

SWANK, R. L. and PUTNAM, T. J. (1943). Amyotrophic lateral sclerosis and related conditions. Archives of Neurology \& Psychiatry, 49, 151-177.

WECHSLER, I. S. and BRODY, S. (1946). The problem of primary lateral sclerosis. Journal of the American Medical Association, 130, 1195-1198. 